\title{
Dietary gamma-linolenic acid supports arachidonic acid accretion and associated $\Delta-5$ desaturase activity in feline uterine but not ovarian tissues*
}

\author{
Amy J. Chamberlin ${ }^{1,2} \ddagger$ and John E. Bauer ${ }^{1,2 \dagger * *}$ \\ ${ }^{1}$ Department of Small Animal Clinical Sciences, Companion Animal Nutrition Research Laboratory, College of Veterinary Medicine and Biomedical \\ Sciences, Texas A\&M University, College Station, TX 77843-4474, USA \\ ${ }^{2}$ Intercollegiate Faculty of Nutrition, Texas A\&M University, College Station, TX 77843-4474, USA
}

(Received 1 January 2013 - Final revision received 2 March 2014 - Accepted 21 March 2014)

Journal of Nutritional Science (2014), vol. 3, e43, page 1 of 4

doi:10.1017/jns.2014.41

\section{Abstract}

Arachidonic acid (ARA) is essential in felines because conversion of dietary linoleic acid (LA) to ARA is rate-limited by low $\Delta 6$-desaturase. Dietary $\gamma$-linolenic acid (GLA) may serve as an ARA precursor by-passing this initial rate-limiting step. This possibility was investigated using twenty-six adult female domestic shorthair cats divided into three groups and fed on complete and balanced diets containing high GLA (GL), high LA (HL) or low LA (LL, control) diets, for $300 \mathrm{~d}$ prior to ovariohysterectomy. Plasma was obtained $1-2 \mathrm{~d}$ before surgery and uterine, ovarian and associated adipose tissues were reserved for lipid analysis. Fatty acid profiles of the plasma phospholipid (PL) fractions and adipose lipids were performed. In the GL group, plasma and uterine tissue PL were significantly enriched in GLA, di-homo GLA (DGLA) and ARA compared with control. However, ovarian and adipose tissue PL were only enriched in DGLA. Enrichment of uterine tissues with DGLA and ARA probably supplies the essential eicosanoid precursors for reproduction when GLA is fed consistently with an active $\Delta 5$-desaturase in uterus. By contrast, this enzyme appears less active in ovary because ARA was not higher compared with control. Earlier reports concluded that ARA was not necessary for fertilisation (an ovarian function), but required for successful pregnancy and reproduction (a uterine function). Adipose tissue DGLA may be a reservoir for ARA synthesis by other tissues upon mobilisation. Dietary GLA may meet feline ARA requirements in the absence of an animal-based preformed source of ARA.

Key words: Feline nutrition: Reproduction: Arachidonate: $\Delta-5$ Desaturase

Feline lipid metabolism is unique among animal species. Most mammalian species convert linoleic acid (LA, $18: 2 n-6)$ to arachidonic acid (ARA, 20:4n-6) via a cascade of enzymatic steps, including $\Delta 6$-desaturation, chain elongation and $\Delta 5$-desaturation ${ }^{(1)}$. Nevertheless, earlier studies determined that cats seemed to lack sufficient $\Delta 6$-desaturase activity and that ARA was a necessary dietary essential for feline species $^{(2-4)}$. Later on, using the stable isotopes, detectible amounts of both $\Delta 6$ - and $\Delta 5$-desaturase products in feline liver were found $^{(5)}$, but it remained to be determined whether ARA synthesis was sufficient for all life stages. Where reproduction is concerned, cats fed an ARA-free diet containing hydrogenated coconut oil found that males did not require ARA for growth or reproduction ${ }^{(6)}$. Females, fed similar diet, were also able to grow, enter oestrus and conceive. However, after conception they produced only a few viable kittens ${ }^{(7)}$ It was concluded that ARA and possibly other PUFA are needed to complete normal full-term pregnancies.

Abbreviations: ARA, arachidonic acid; DGLA, di-homo $\gamma$-linolenic acid; GL, high $\gamma$-linolenic acid; GLA, $\gamma$-linolenic acid; HL, high linolenic acid; LA, linoleic acid; LL, low linolenic acid; PL, phospholipid.

†Corresponding author: Dr Bauer, email jbauer@cvm.tamu.edu

* This article was published as part of the WALTHAM International Nutritional Sciences Symposium Proceedings 2013.

$\ddagger$ Present address: Oxbow Animal Health, 29012 Mill Road, Murdoch, NE 68407, USA.

** Present address: Professor Affiliate, Colorado State University, 1678 Campus Delivery, Fort Collins, CO 80523-167828, USA (email jbauer@cvm.tamu.edu). 
A recent study of plasma phospholipid (PL) fatty acids from our laboratory found evidence for $\Delta 5$-desaturation in feline species fed dietary GLA ${ }^{(8)}$. However, in view of the earlier findings regarding feline reproduction, it was subsequently hypothesised that feeding cats a GLA-enriched diet by-passes the $\Delta 6$-desaturase in uterine and ovarian tissues thereby providing precursors for ARA synthesis in these tissues.

\section{Experimental methods}

The present investigation was approved by the Texas A\&M University Animal Care and Use Committee. Twenty-six, sexually intact female cats ranging between $1 \frac{1}{2}$ and 2 years of age were used in the study. They had been carefully fed ad libitum over a $300 \mathrm{~d}$ period on the diets employed in the present study according to their metabolic body weights using the equation, Daily Metabolisable Energy $=418.4 \mathrm{~kJ}(100 \mathrm{kcal})$ $\mathrm{ME} \times \mathrm{W}_{\mathrm{kg}}^{0.67}$ per $\mathrm{d}$ with water. Daily consumption records and weekly body weights were recorded. Body condition scores using a 1 to 9 scale, where 5 is ideal, were evaluated exclusively by one of the investigators and used to adjust amounts fed. In this way, a range of body condition score values between 5 and 6 was achieved with a median value of 5 over the entire feeding period. Body weights of the cats ranged from 2.4 to $3.9 \mathrm{~kg}$ with a median value of 3.29 . All cats were housed individually at the Laboratory Animal Research Resources facility, Texas A\&M University, according to the American Physiological Society Guidelines for Animal Research and guidelines set forth by Texas A\&M University Care and Use Committee. Each cage for cats was $2.5 \mathrm{~m}$ long, $2.0 \mathrm{~m}$ high and $1.2 \mathrm{~m}$ wide. Prior to the investigation and at intervals throughout the study physical examinations, complete blood counts, serum biochemistry profiles and $\mathrm{T}_{3}$, thyroid-stimulating hormone assays were performed on all cats to verify their normal clinical status.

The cats were cared for by the Laboratory Animal Research and Resources staff and resident veterinarian. Members of the Companion Animal Nutrition Laboratory monitored and fed the cats daily. These protocols were approved by Texas A\&M University Animal Care and Use Committee. Previously, the animals had been randomly assigned to one of three diet groups for a separate project lasting $56 \mathrm{~d}$. They were then continued on their respective diets thereafter for $300 \mathrm{~d}$ for the present study ${ }^{(8)}$. Twenty-nine cats had been originally randomised using a computer spreadsheet randomisation function (Microsoft ${ }^{\circledR}$ Excel 2008), but three were excluded prior to $300 \mathrm{~d}$ for non-dietary related concerns. The earlier study revealed suitable statistical power for plasma PL fatty acid profile differences using 8-10 cats per group when fed carefully on controlled diets ${ }^{(8)}$. In addition, plasma PL are generally considered to reflect tissue levels under steady-state conditions and this condition would be likely after $300 \mathrm{~d}$ of feeding. Thus, the sample size of the present study was considered to be adequately powered to detect tissue differences. The diets in the present study were complete and balanced containing either high $\gamma$-linolenic acid (GL, $n$ 10), high linoleic acid (HL, $n$ 7) or low linoleic acid (LL, $n$ 9, control), for a total of $300 \mathrm{~d}$ prior to ovariohysterectomy. The HL diet used LA-rich safflower oil and
Table 1. Experimental diet nutrient and PUFA profiles ( $\mathrm{g} / \mathrm{kg} \mathrm{DM}$ )

\begin{tabular}{|c|c|c|c|}
\hline \multirow{2}{*}{ Diet component } & \multicolumn{3}{|c|}{ Diet } \\
\hline & GL & $\mathrm{HL}$ & LL (control) \\
\hline & $\mathrm{g} / \mathrm{kg}$ DM & $\mathrm{g} / \mathrm{kg}$ DM & $\mathrm{g} / \mathrm{kg}$ DM \\
\hline Crude protein & 346 & 355 & 349 \\
\hline Fat & 185 & 199 & 182 \\
\hline Ash & 71 & 77 & 70 \\
\hline Fibre & 21 & 18 & 20 \\
\hline \multicolumn{4}{|l|}{ PUFA } \\
\hline \multicolumn{4}{|l|}{$n-6$} \\
\hline LA $(18: 2 n-6)$ & $20 \cdot 0$ & $65 \cdot 8$ & $18 \cdot 7$ \\
\hline GLA $(18: 3 n-6)$ & 3.8 & 0.2 & $<0.1$ \\
\hline ARA $(20: 4 n-6)$ & 0.30 & 0.31 & 0.31 \\
\hline \multicolumn{4}{|l|}{$n-3$} \\
\hline ALA $(18: 3 n-3)$ & 1.2 & 1.8 & 1.3 \\
\hline \multirow{2}{*}{ Other $n-3$} & $<0.1$ & $<0.1$ & $<0.1$ \\
\hline & $\%$ & $\%$ & $\%$ \\
\hline \multirow[t]{2}{*}{ Moisture } & $7 \cdot 7$ & 8.7 & 8.4 \\
\hline & kJ/kg & kJ/kg & kJ/kg \\
\hline Energy & $18480 \cdot 7$ & $18058 \cdot 1$ & $18062 \cdot 3$ \\
\hline
\end{tabular}

the LL diet contained coconut oil in place of safflower oil to reduce LA to low, but adequate, levels. The GL diet was enriched with high GLA borage oil containing $70 \%$ GLA plus an adequate amount of LA. The three diets were comparable in all respects except for omega-6 fatty acid type, while the omega- 3 contents of all diets were similar and kept to a minimum by design (Table 1). The nutrient composition and major PUFA contents of the diets were determined (Nestlé Purina Laboratories) and found to be within the expected analytical variance for the formulated targets of 350 (SD 15) $\mathrm{g} / \mathrm{kg}$ DM for protein, 190 (SD 27) g/ kg DM for fat, 75 (SD 11) g/ kg DM for ash and 20 (sD 5) g/ kg/DM for fibre (Table 1).

Cats were spayed after $300 \mathrm{~d}$ of feeding using the standard surgical techniques. Plasma samples were collected into tubes containing EDTA as anticoagulant $1-2 \mathrm{~d}$ before surgery, and ovaries, uterus and adjacent adipose tissues were harvested, trimmed of excess connective tissues and frozen at $-80^{\circ} \mathrm{C}$ for the subsequent analysis. Total lipids were extracted from tissue homogenates prepared in phosphate-buffered saline ( $\mathrm{pH} 7.4)$ and plasma. PL were fractionated from plasma, ovarian and uterine tissues and total the lipids were extracted from adipose tissues. Fatty acid methyl esters of the extracts were prepared and analysed via capillary $\mathrm{GC}^{(9)}$.

All data are presented as $\mathrm{mol} / 100 \mathrm{~mol}$ of fatty acids and SD and evaluated using SPSS 16.0 for Windows (SPSS Inc, Chicago, IL). To determine whether the data were normally distributed, the Shapiro-Wilks test was performed. One-way ANOVA was utilised when data appeared normally distributed for diet comparisons. Where significant diet group differences were found, Tukey multiple comparison tests were performed $(P<0 \cdot 5)$. Non-normally distributed data were analysed using the Kruskal-Wallis test. All results were considered statistically significant if the two-tailed $P$ value was $<0 \cdot 05$.

\section{Results}

Significant increases in plasma PL di-homo GLA (DGLA) and ARA were observed with the GL diet $(P=0.009$ and 0.007, respectively), while the HL and LL diets resulted in lower, 
Table 2. Major PUFA of feline tissues when diets after $300 \mathrm{~d}$ of feeding ( $\mathrm{mol} / 100 \mathrm{~mol})$

\begin{tabular}{|c|c|c|c|c|c|c|}
\hline \multirow{2}{*}{ Tissue } & \multicolumn{2}{|c|}{ GL diet } & \multicolumn{2}{|c|}{ HL diet } & \multicolumn{2}{|c|}{ LL diet (control) } \\
\hline & Mean or (median) & SD or (range) & Mean or (median) & SD or (range) & Mean or (median) & SD or (range) \\
\hline \multicolumn{7}{|c|}{ LA $(18: 2 n-6)$} \\
\hline Plasma & $21.5^{\mathrm{a}}$ & $2 \cdot 8$ & $35.7^{\mathrm{b}}$ & 1.9 & $28.5^{\mathrm{c}}$ & 1.9 \\
\hline Uterus & $8 \cdot 6^{\mathrm{a}}$ & 0.3 & $16 \cdot 8^{\mathrm{b}}$ & 0.8 & $13 \cdot 9^{c}$ & $1 \cdot 1$ \\
\hline Ovary & $7 \cdot 7$ & $7 \cdot 7$ & 9.6 & 4.6 & 8.7 & $2 \cdot 6$ \\
\hline Adipose & $16 \cdot 2^{\mathrm{a}}$ & 0.7 & $32 \cdot 3^{b}$ & 0.9 & $15 \cdot 2^{\mathrm{a}}$ & 0.5 \\
\hline \multicolumn{7}{|c|}{ GLA (18: $3 n-6)$} \\
\hline Plasma & $0.6^{a}$ & 0.3 & $0.2^{\mathrm{b}}$ & 0.1 & $0.3^{\mathrm{b}}$ & 0.1 \\
\hline Uterus & $(0.0)$ & $(0.0-0.2)$ & $(0.0)$ & $(0.0-0.1)$ & $(0.0)$ & $(0.0-0.7)$ \\
\hline Ovary & 0.4 & 0.6 & 0.4 & 0.8 & 0.3 & 0.6 \\
\hline Adipose & $(0.6)^{a}$ & $(0.04-0.7)$ & $(0.0)^{b}$ & $(0.0-0.1)$ & $(0.0)^{b}$ & $(0.0-0.6)$ \\
\hline \multicolumn{7}{|c|}{ DGLA (20 : 3n-6) } \\
\hline Plasma & $10 \cdot 6^{\mathrm{a}}$ & 4.2 & $0.8^{\mathrm{b}}$ & 0.3 & $1.9^{b}$ & 0.2 \\
\hline Uterus & $4 \cdot 0^{\mathrm{a}}$ & 0.9 & $1 \cdot 2^{\mathrm{b}}$ & 0.5 & $1.5^{\mathrm{b}}$ & 0.5 \\
\hline Ovary & $4.0^{\mathrm{a}}$ & $1 \cdot 1$ & $1.9^{\mathrm{b}}$ & 1.4 & $2 \cdot 0^{\mathrm{b}}$ & $1 \cdot 1$ \\
\hline Adipose & $0.6^{\mathrm{a}}$ & 0.2 & $0.06^{b}$ & 0.08 & $0.12^{b}$ & 0.08 \\
\hline \multicolumn{7}{|c|}{ ARA $(20: 4 n-6)$} \\
\hline Plasma & $5 \cdot 3^{\mathrm{a}}$ & 0.9 & $2 \cdot 2^{\mathrm{b}}$ & 0.6 & $4 \cdot 1^{\mathrm{c}}$ & 0.4 \\
\hline Uterus & $15 \cdot 8^{a}$ & 0.7 & $10 \cdot 3^{b}$ & 0.8 & $12 \cdot 1^{b}$ & 0.7 \\
\hline Ovary & $12 \cdot 6$ & 3.0 & $10 \cdot 0$ & $2 \cdot 3$ & 11.5 & 3.3 \\
\hline Adipose & $0.20^{\mathrm{a}}$ & 0.05 & $0.07^{\mathrm{b}}$ & 0.03 & $0.15^{a, b}$ & 0.3 \\
\hline
\end{tabular}

Values are mean and SD for parametric data, whereas those in parentheses are median and range of values for non-parametric data. GL diet $(n 10)$, HL diet $(n 7)$ and LL diet $(n 9)$; superscript letters not in common in a row indicate statistically significant differences among diet groups; $P<0.05$.

and similar, amounts of DGLA (Table 2). It is of interest that plasma PL ARA was significantly lowest and plasma PL LA was significantly highest with the HL diet, whereas the GL diet was lowest and the LL (control) diet intermediate in value for each of these fatty acids (Table 2). It appeared that a relative enrichment of the plasma PL fraction with LA had occurred at the expense of ARA when the HL diet was fed. Similar to plasma, uterine tissue homogenate PL had statistically significantly higher amounts of DGLA and ARA with the GL diet $(P<0.001$ in both cases), but GLA was not different compared with the other diet groups. Uterine LA was again significantly higher with the HL diet compared with the GL and LL (control) diets $(P=0 \cdot 005)$. In this case, unlike plasma, uterine ARA of cats fed HL and LL diets were similar.

A noteworthy finding from the present study was that ovarian tissue PL accumulated DGLA ( $P=0.004)$, but no additional ARA with the GL diet compared with the other two diets. No other differences among the PUFA were observed in this ovarian tissue fraction. Adipose tissue lipid extracts revealed small, yet statistically significant enrichment of GLA and DGLA with the GL diet $(P<0.001$ in both cases) with a marked accumulation of LA again observed with the HL diet $(P<0.03)$ (Table 2).

\section{Discussion}

The accumulation of ARA, its DGLA precursor, and corresponding decrease of LA in plasma and uterine homogenate PL fractions of cats fed the GLA-enriched diet $v$. the other two diets was not unexpected. These findings confirm the results from our earlier study of plasma PL of cats fed these same three diets during a $56-\mathrm{d}_{\text {period }}{ }^{(8)}$ and extend them to include fatty acid compositions of feline reproductive tissues and evidence for active $\Delta 5$-desaturation in uterine tissue.
The uterine accretion of ARA with the GL diet would be expected to provide the substrate for PG synthesis in support of the successful full-term pregnancy ${ }^{(10)}$. Thus, a diet containing GLA from vegetable sources may be possible to meet feline ARA requirements for reproduction in the absence of a pre-formed animal-based ARA source.

By contrast, the lack of additional ARA accumulation in ovarian homogenate PL with dietary GLA was somewhat unexpected although DGLA was observed. This result suggests a low or absent of $\Delta 5$-desaturation in the ovarian tissue. Thus, in order for this tissue to become enriched with ARA it is likely to be the result of circulatory transport of ARA that has been either synthesised in other tissues or from direct dietary sources. This finding is consistent with the earlier reports by MacDonald et al. and by Morris who noted that dietary ARA did not seem to be needed for fertilisation to occur (an ovarian function), but necessary for successful pregnancy and viable kittens (a uterine function) ${ }^{(6,7)}$. Also, modest but statistically significant higher amounts of adipose GLA and DGLA when GLA is fed may provide a reservoir for tissues that possess active $\Delta 5$-desaturation allowing subsequent ARA synthesis.

Finally, it is noteworthy that higher amounts of LA were observed in plasma and uterine PL at the expense of ARA with high dietary LA (HL diet). This phenomenon was presumably due to competition between these two PUFA for incorporation into the PL fractions. Although it is unknown what level of ARA enrichment of tissue PL may be needed for optimal eicosanoid production in cats overall, the possibility exists that some threshold amount of ARA may be needed for optimal cell function. If so, the amount of LA used in the HL diet of the present study may help define a safe upper limit for dietary LA concentration in cats because high dietary amounts may effectively limit incorporation of ARA in the PL fractions. 


\section{Acknowledgements}

We thank K. E. Bigley for sampling assistance. A. J. C. and J. E. B. designed research; A. J. C. and K. E. Bigley conducted research; A. J. C. analysed data; J. E. B. and A. J. C. authored the manuscript; and J. E. B. had primary responsibility for final content. All authors read and approved the final manuscript. Funding provided by the Mark L. Morris Professorship in Clinical Nutrition, Texas A\&M University, College Station, TX, USA. There were no conflicts of interest among authors. The work was presented in abstract form at the 2013 Waltham International Nutrition Sciences Symposium, Portland OR, USA, September 30-October 4.

This paper was published as part of the WALTHAM International Nutritional Sciences Symposium Proceedings 2013, publication of which was supported by an unrestricted educational grant from Mars Incorporated. The papers included in these proceedings were invited by the Guest Editor and have undergone the standard journal formal review process. They may be cited.

\section{References}

1. Sinclair AJ, McLean JG \& Monger EA (1979) Metabolism of linoleic acid in the cat. Lipids 14, 932-936.
2. Rivers JPW, Sinclair AJ \& Crawford MA (1975) Inability of the cat to desaturate essential fatty acids. Nature 258, 171-173.

3. Rivers JPW, Hassam AG, Crawford MA \& Brambell MR (1976) The absence of $\Delta 6$-desaturase activity in cats. Proc Nutr Soc 35, 69A.

4. Rivers JPW, Sinclair AJ, Moore DP \& Crawford MA (1976) The abnormal metabolism of essential fatty acids in cat. Proc Nutr Soc 35, 68A.

5. Pawlosky RA, Barnes A \& Salem N Jr (1994) Essential fatty acid metabolism in the feline: relationship between liver and brain production of long chain polyunsaturated fatty acids. J Lipid Res 35, 2032-2040.

6. MacDonald ML, Rogers QR, Morris JG \& Cuppsaz PT (1984) Efflects of linoleate and arachidonate deficiencies on reproduction and spermatogenesis in the cat. J Nutr 114, 719-726.

7. Morris JG (2004) Do cats need arachidonic acid for reproduction? J Anim Physiol Anim Nutr (Berl) 88, 131-137.

8. Trevizan L, de Mello Kessler A, Brenna JT, Lawrence P, Waldron MK \& Bauer JE (2012) Maintenance of arachidonic acid and evidence of $\Delta 5$ desaturation in cats fed $\gamma$-linolenic and linoleic acid enriched diets. Lipids 47, 413-423.

9. Dunbar BL, Bigley KE \& Bauer JE (2010) Early and sustained enrichment of serum n-3 long chain polyunsaturated fatty acids in dogs fed a flaxseed supplemented diet. Lipids 45, 1-10.

10. Kumar R, Ramteke PW, Nath A, Pramod RK, Singh SP, Sharma SK \& Kumar S (2013) Role of candidate genes regulating uterine prostaglandins biosynthesis for maternal recognition of pregnancy in domestic animals. ISRN Physiology, Hindawi Publishing, Volume 2013, Article ID 854572, 8 pp. http://dx.doi.org/10. $1155 / 2013 / 854572$ 